\title{
Efficacy and safety of first-line treatments with immune checkpoint inhibitors plus chemotherapy for non-squamous non-small cell lung cancer: a meta-analysis and indirect comparison
}

\author{
Yafei Shi", Wei Chen", Chunyu Li, Yujun Zhang, Mingming Bo, Shuya Qi, Guohui Li \\ Department of Pharmacy, National Cancer Center/National Clinical Research Center for Cancer/Cancer Hospital, Chinese Academy of Medical \\ Sciences and Peking Union Medical College, Beijing, China \\ Contributions: (I) Conception and design: Y Shi, W Chen, G Li; (II) Administrative support: G Li; (III) Provision of study materials or patients: Y Shi, \\ W Chen, C Li, Y Zhang, M Bo, S Qi; (IV) Collection and assembly of data: C Li, Y Zhang, M Bo, S Qi; (V) Data analysis and interpretation: Y Shi, \\ W Chen; (VI) Manuscript writing: All authors; (VII) Final approval of manuscript: All authors. \\ \#These authors contributed equally to this work. \\ Correspondence to: Guohui Li. Department of Pharmacy, National Cancer Center/National Clinical Research Center for Cancer/Cancer Hospital, \\ Chinese Academy of Medical Sciences and Peking Union Medical College, 17 Panjiayuannanli, Beijing 100021, China. \\ Email: lgh0603@cicams.ac.cn.
}

Background Recently, several clinical studies have evaluated the first-line use of immune checkpoint inhibitors (ICIs) combined with platinum-doublet chemotherapy in patients with non-squamous non-small cell lung cancer (NSCLC), however, the differences in safety and efficacy between the various types of ICIs still require investigation. In this study, we evaluated the efficacy and safety of the first-line use of ICIs combined with platinum-doublet chemotherapy in patients with non-squamous NSCLC by meta-analysis and indirect comparison.

Methods: Literature searches were performed using PubMed, the Cochrane Library, Embase, China Knowledge Resource Integrated Database, and Wanfang Data to identify all relevant randomized clinical trials for non-squamous NSCLC after 2010. Overall survival (OS), progression-free survival (PFS), and adverse effects (AEs) were pooled for meta-analysis and indirect comparison. Subgroup analyses were conducted to examine the factors associated with PFS.

Results: The meta-analysis showed that the additional use of ICIs could significantly improve PFS and OS. The indirect comparison showed no significant difference in pembrolizumab + chemotherapy and atezolizumab + chemotherapy in the reducing of disease progression, while a significant difference in restricted mean survival time (RMST) was found between pembrolizumab + chemotherapy compared with atezolizumab + chemotherapy. A significant increase in grade $\geq 3$ AEs was observed with the additional use of atezolizumab combined with chemotherapy. Subgroups including PD-1 status [high ( $>50 \%)$, intermediate (1-49\%), and negative (<1\%) expression], sex (male and female), smoking status (current or former smoker, and never smoked), liver metastases (with and without), age ( $>65$ and $\leq 65$ ) and Eastern Cooperative Oncology Group (ECOG) score $(\mathrm{ECOG}=0$ and $\mathrm{ECOG}=1)$ were all associated with better PFS.

Conclusions: This meta-analysis confirmed the treatment effects of ICIs combined with chemotherapy for non-squamous NSCLC. The pembrolizumab combination group had a greater RMST benefit compared with the atezolizumab combination group. Furthermore, our study also demonstrated a PFS advantage for non-squamous NSCLC using ICIs combined with chemotherapy irrespective of programmed death-ligand 1 (PD-L1) expression level, smoking status, liver metastasis status, sex, age and ECOG score. Due to the significant increase in AEs (> grade 3), more attention should be paid to the additional use of atezolizumab.

Keywords: Non-squamous non-small cell lung cancer (non-squamous NSCLC); immune checkpoint inhibitors (ICIs); first-line; efficacy; safety 
Submitted Jul 28, 2020. Accepted for publication Dec 08, 2020.

doi: 10.21037/apm-20-1498

View this article at: http://dx.doi.org/10.21037/apm-20-1498

\section{Introduction}

Worldwide, lung cancer remains the major cause of cancer death (1). Non-small cell lung cancer (NSCLC) accounts for approximately $85 \%$ of all primary lung cancers, of which, nearly two-thirds of patients are diagnosed with the non-squamous histological subtype (2). Currently, platinum-doublet chemotherapy is the standard first-line treatment for patients with non-squamous NSCLC in the absence of epidermal growth factor receptor (EGFR) or anaplastic lymphoma kinase (ALK) aberrations, as molecular targeted therapy cannot be achieved in these patients (3), immune checkpoint inhibitors (ICIs), such as inhibitors of programmed cell death protein 1 (PD-1) and its ligand (PDL1), can block the combination of PD-1 and PD-L1 in T cells and cancer cells, preventing tumor immunoevasion, and recovering the function of $\mathrm{T}$ cells for killing cancer cells (4). PD-1 and PDL-1 inhibitors have promoted the development of treatments for lung cancer (5-8). To date, a series of trials including CheckMate 017, CheckMate 057, KEYNOTE-010, and OAK have confirmed the benefit of these ICIs for decreasing the mortality risk of advanced NSCLC in the second-line setting (9). In recent studies, increasing evidence suggests that the immunological processes also have important anti-tumor activities in chemotherapy (10-12), thus, the combination of ICIs therapy and chemotherapy may be synergistic in improving antitumour activity. Several clinical studies have already evaluated the first-line use of ICIs combined with platinum-doublet chemotherapy in patients with non-squamous NSCLC. Superior overall survival (OS) and progression-free survival (PFS) were demonstrated with the addition use of ICIs in several previous studies (13-17). However, the differences in efficacy and safety between the various types of ICIs are still unclear in the first-line treatment of non-squamous NSCLC. The factors associated with improvements in PFS have been controversial in different trials. Also, some of the median OS rates have not been reached in published studies, and some of the studies might have violated the proportional hazards assumption when the hazard ratios (HRs) vary with time (18), suggesting that conventional measures of survival benefit have clinical limitations. Therefore, a meta-analysis and indirect comparison of the safety and efficacy of the additional use of ICIs in non-squamous NSCLC is urgently needed. Also, to reduce potential variability in survival benefit using conventional measures, an indirect comparison with a robust measure is needed.

Recently, restricted mean survival time (RMST), a robust summary measure which can measure the area under the survival curve up to a restricted time point, was confirmed to be a viable alternative approach that could overcome the limitations of conventional measures $(19,20)$. Therefore, in this study, we performed a meta-analysis and indirect comparison measured with RMST in accordance with the following preferred reporting items for systematic reviews and meta-analyses (PRISMA) reporting checklist (available at http://dx.doi. org/10.21037/apm-20-1498) to evaluate the efficacy and safety of first-line use of ICIs in combination with platinum-doublet chemotherapy in patients with non-squamous NSCLC (21).

\section{Methods}

\section{Search strategy}

A literature search was performed using PubMed, the Cochrane Library, Embase, China Knowledge Resource Integrated Database, and Wanfang Data to identify all relevant randomized clinical trials for non-squamous NSCLC after 2010. Furthermore, the reference lists of all available original studies, reviews, and meeting reports from the main international meetings for lung cancer were also manually searched. The following keywords were used for searching: "non-small cell lung cancer", "nonsquamous lung cancer", "nivolumab", "pembrolizumab", "atezolizumab", "PD-1 inhibitor", and "PD-L1 inhibitor". The language of studies was restricted to English. Three authors independently completed the search, and discrepancies were resolved by discussion.

\section{Selection criteria}

Following the patient, intervention, comparison, outcome 
(PICO) framework, studies had to meet the following criteria: (I) patients with previously untreated metastatic non-squamous NSCLC; (II) studies compared the additional use of a PD-1 inhibitor or PD-L1 inhibitor with platinum-doublet chemotherapy in the first-line setting; (III) reported outcomes included one or more of the following: OS, PFS, toxicity; (IV) studies were prospective randomized controlled trials (RCTs).

\section{Data extraction}

The following data from the eligible studies were extracted, if available: (I) patient characteristics including age, sex, and tumor stage; (II) treatment characteristics, including treatment history, treatment regimens, and treatment cycles; (III) outcomes, including OS, PFS, follow-up duration, and adverse events; (IV) trial characteristics, including trial phase and the number of patients in each group; (V) publication characteristics, including publication authors and years of publication.

\section{Quality assessment}

The quality of included studies was assessed using the Cochrane Collaboration's risk of bias tool (22). Six key domains in the Cochrane Collaboration's risk of bias tool were evaluated: random sequence generation, allocation concealment, blinding of participants, personnel and outcome assessors, incomplete outcome data, selective outcome reporting, and other sources of bias. Each domain was assigned to a risk status with low, medium, high, or unclear.

\section{Statistical analysis}

OS and PFS were expressed as a HR, and AEs were expressed as an odds ratio (OR). Review Manager (RevMan 5.3) software was used to calculate the pooled effect of HR [with a $95 \%$ confident interval (CI)] and OR (with $95 \%$ CI). Treatment regimens conducted subgroup analyses in the intervention group (PD-1 inhibitor and PD-L1 inhibitor). A $\mathrm{P}$ value of $<0.05$ was considered significant. Further subgroup analyses by PD-L1 expression, sex, age, liver metastatic status, and Eastern Cooperative Oncology Group (ECOG) score were also conducted to examine PFS's factors. Heterogeneity between studies was tested using the $\chi^{2}$ test and $\mathrm{I}^{2}$ statistics. $\mathrm{P}$ value for $\chi^{2}<0.1$ or an $\mathrm{I}^{2}$ statistic $>50 \%$ was considered statistically significant for heterogeneity, and the random-effects model was used to calculate the pooled estimate in this situation. Otherwise, the fixed effect model was used. Additionally, for studies that enrolled similar patients with similar control chemotherapy regimens, an indirect comparison or network meta-analysis was performed to compare the treatment effects among the different ICIs. A link of similar chemotherapy regimen compared the treatment effects. For the indirect comparison, the following formulas was used as previously described (23): $\log H R_{A B}=\log H_{A C}-\log H R_{B C}$, where $\log \mathrm{HR}_{\mathrm{AB}}$ represents $\log \mathrm{HR}$ of the adjusted indirect comparison for arm A vs. arm $\mathrm{B}, \log \mathrm{HR}_{\mathrm{AC}}$ represents $\log \mathrm{HR}$ of the direct comparison for arm A vs. arm C, and $\log \mathrm{HR}_{\mathrm{BC}}$ represents $\log \mathrm{HR}$ of the direct comparison for arm B vs. arm C. The standard error (SE) was estimated by $\mathrm{SE}\left(\log H \mathrm{R}_{\mathrm{AB}}\right)=\sqrt{S E\left(\log H R_{A C}\right)^{2}+S E\left(\log H R_{B C}\right)^{2}}$. The RMST was used as a supplementary endpoint when the reported follow-ups were not the same. $\mathrm{R}$ version 3.6.0 (R Project for Statistical Computing) was used to complete the indirect comparison.

\section{Results}

\section{Eligible studies}

Up to July 12,2020 , a total of 1,238 articles were identified. Finally, 5 studies matching the study inclusion criteria were identified. The study identification and selection process is shown in Figure 1. Trial characteristics of the eligible studies are list in Table 1.

\section{Quality of studies}

Since most of the included studies were open-label, detection bias, reporting bias, and other bias were all or mostly in the low-risk category. The risk of attrition bias was low in IMPOWER 130, while the others studies were unclear as the trials' final results were not reported. The information for determining selection and performance bias was insufficient except for KEYNOTE-189, and these 2 items for most of the included studies were all assigned to a risk status of unclear. The risk of bias graph and summary are shown in Figure 2.

\section{Pooled analysis of the OS and PFS}

The comparison results of PFS and OS for the included trials were all reported. The pooled HR for PFS was 0.58 


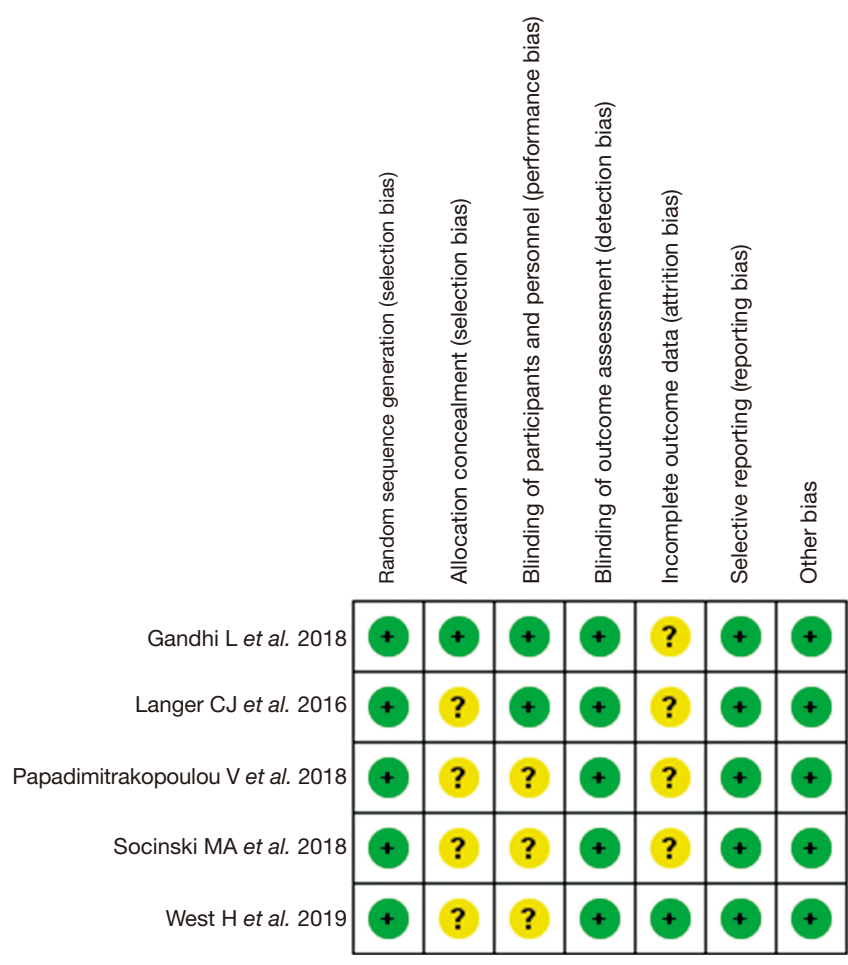

Figure 1 Study identification and selection process.

(0.52-0.64), showing a significant improvement in both the PD-1 inhibitor + chemotherapy $\left[0.49(0.41-0.58), \mathrm{I}^{2}=0\right]$ and PD-L1 inhibitor + chemotherapy [0.61 $\left.(0.56-0.68), \mathrm{I}^{2}=0\right]$ subgroups (Figure 3). As for OS, the pooled HR was 0.72 (0.61-0.84). A significant lower risk of death was seen in both the PD-1 inhibitor + chemotherapy [0.56 (0.46-0.69), $\left.\mathrm{I}^{2}=0\right]$ and PD-L1 inhibitor + chemotherapy [0.79 (0.700.90), I2=0] subgroups (Figure 4).

\section{Indirect comparison of PFS}

Chemotherapy combined with pemetrexed + cisplatin as a comparison was used in both KEYNOTE-189 and Impower-132. In these 2 trials, the PFS data was already matured, thus, we compared the relative effects for PFS. As a result, indirect comparison using the frequency method showed no significant difference between pembrolizumab + chemotherapy and atezolizumab + chemotherapy in the reducing of disease progression $\left[\mathrm{HR}_{\text {indirect }}=0.80(0.61-1.04)\right]$.

\section{Indirect comparison of RMST for OS}

The reported follow-ups for OS in KEYNOTE-189 and
IMpower-132 were not the same, thus, we reconstructed the OS curve from the published data, and the differences in RMST at 18 months were compared. As a result, the differences in RMST were 2.781 (1.751-3.812) months with pembrolizumab + chemotherapy compared with chemotherapy, and $1.153(0.119-2.187)$ months with atezolizumab + chemotherapy compared with chemotherapy (Figure 5). The indirect comparison showed that the difference between pembrolizumab + chemotherapy and atezolizumab + chemotherapy was $1.63(0.17-3.09)$ months.

\section{Factors associated with PFS}

The factors associated with PFS are listed in Table 2. Subgroups including PD-1 status [high (>50\%), intermediate $(1-49 \%)$, and negative $(<1 \%)$ expression], sex (male and female), smoking status (current or former smoker and never smoked), liver metastases (with and without), age ( $>65$ and $\leq 65)$, and ECOG score $(\mathrm{ECOG}=0$ and $\mathrm{ECOG}=1$ ) were all associated with better PFS.

\section{Grade $\geq 3$ AEs}

Grade $\geq 3$ AEs were detected between the ICIs + chemotherapy group and the chemotherapy group alone. In the 2 ICIs + chemotherapy regimens, there were no significant differences in grade $\geq 3$ AEs between the pembrolizumab + chemotherapy group compared with the chemotherapy group alone $\left[\mathrm{OR}=1.05(0.76-1.45), \mathrm{I}^{2}=0\right.$ ], while significantly more grade $\geq 3$ AEs were observed with the additional use of atezolizumab in combination with chemotherapy [OR $=1.54(1.28-1.86), \mathrm{I}^{2}=0$; Figure 6].

\section{Discussion}

In recent years, clinicians have characterized advanced NSCLC using many driver mutations, such as EGFR, ALK, BRAF and KRAS. Consequently, molecularlyguided therapeutics have been used in patients with advance NSCLC (24). However, it is important to realize that several patients cannot benefit from these therapeutics if these oncogenic drivers are not presence. Hence, platinumbase chemotherapy doublets are still the standard first-line treatment for most advanced NSCLC patients. With the recent advances in immunotherapy, clinicians have realized that the early used of ICIs can confer a greater survival benefit for many solid tumors. Trials for first-line treatment of NSCLC have also been conducted. In the present study, 
Table 1 Characteristics of the eligible studies

\begin{tabular}{|c|c|c|c|c|c|c|}
\hline Study & $\begin{array}{c}\text { Case } \\
\text { numbers }\end{array}$ & $\begin{array}{c}\text { Median age } \\
\text { [range] (years) }\end{array}$ & Male & Randomized arms & $\begin{array}{c}\text { Median PFS } \\
\text { (range) (months) }\end{array}$ & $\begin{array}{c}\text { Median OS (range) } \\
\text { (months) }\end{array}$ \\
\hline Gandhi Let al. 2018 & 616 & $\begin{array}{c}65[34-84] \text { vs. } \\
63.5[34-84]\end{array}$ & $\begin{array}{c}254(62 \%) \text { vs. } \\
109(52.9 \%)\end{array}$ & $\begin{array}{l}\text { Pembrolizumab + pemetrexed } \\
+ \text { cisplatin } v s . \text { placebo }+ \\
\text { pemetrexed + cisplatin }\end{array}$ & $\begin{array}{c}9.0(8.1-9.9) \text { vs. } \\
4.9(4.7-5.5)\end{array}$ & $\begin{array}{c}22.0(19.5-25.2) v s . \\
10.7(8.7-13.6)\end{array}$ \\
\hline Langer CJ et al. 2016 & 123 & $\begin{array}{c}62.5[54-70] \text { vs. } \\
63.2[58-70]\end{array}$ & $\begin{array}{c}22 \text { (37\%) vs. } \\
26(41 \%)\end{array}$ & $\begin{array}{l}\text { Pembrolizumab + carboplatin } \\
+ \text { pemetrexed vs. carboplatin + } \\
\text { pemetrexed }\end{array}$ & $\begin{array}{c}19(8.5-N R) \text { vs. } \\
8.9(6.2-11.8)\end{array}$ & $\begin{array}{c}\text { NR }(22.8-N R) \text { vs. } \\
20.9(14.9-N R)\end{array}$ \\
\hline $\begin{array}{l}\text { Socinski MA et al. } \\
2018\end{array}$ & 1045 & $\begin{array}{c}63[31-89] \text { vs. } \\
63[31-90]\end{array}$ & $\begin{array}{c}240(60 \%) \text { vs. } \\
239(60 \%)\end{array}$ & $\begin{array}{l}\text { Atezolizumab + bevacizumab } \\
+ \text { paclitaxel + carboplatin vs. } \\
\text { bevacizumab + paclitaxel + } \\
\text { carboplatin }\end{array}$ & $\begin{array}{c}8.3(7.7-9.8) \text { vs. } \\
6.8(6.0-7.1)\end{array}$ & $\begin{array}{c}19.2(17.0-23.8) v s . \\
14.7(13.3-16.9)\end{array}$ \\
\hline
\end{tabular}

NR, not reached; OS, overall survival; PFS, progression-free survival.

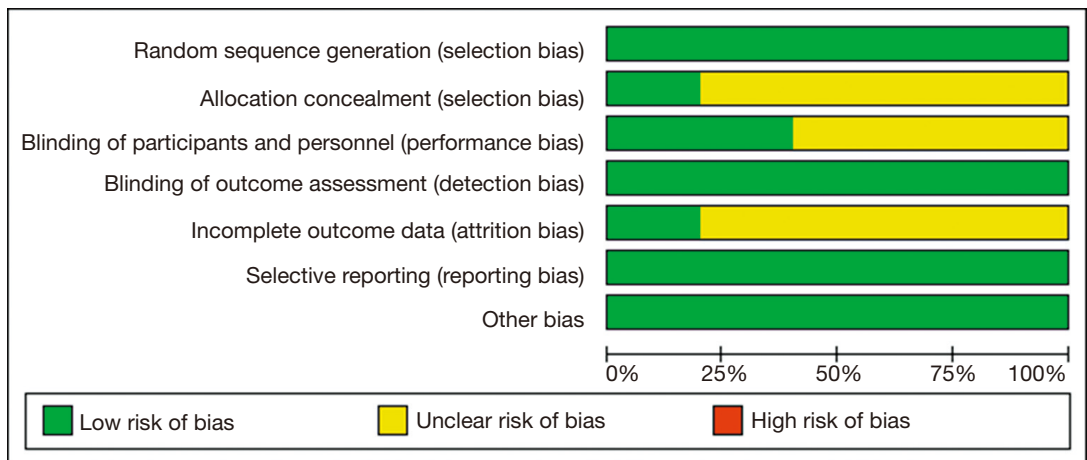

Figure 2 Risk of bias graph and summary.

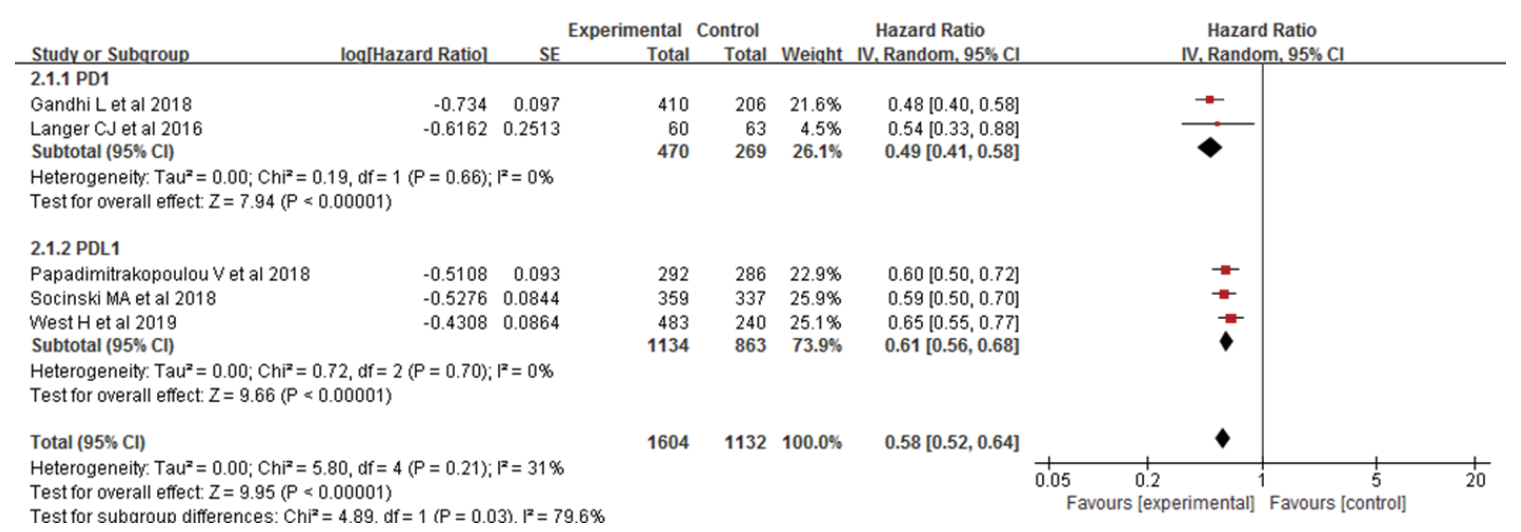

Figure 3 Forest plot of hazard ratios (HR) for progression-free survival (PFS). 


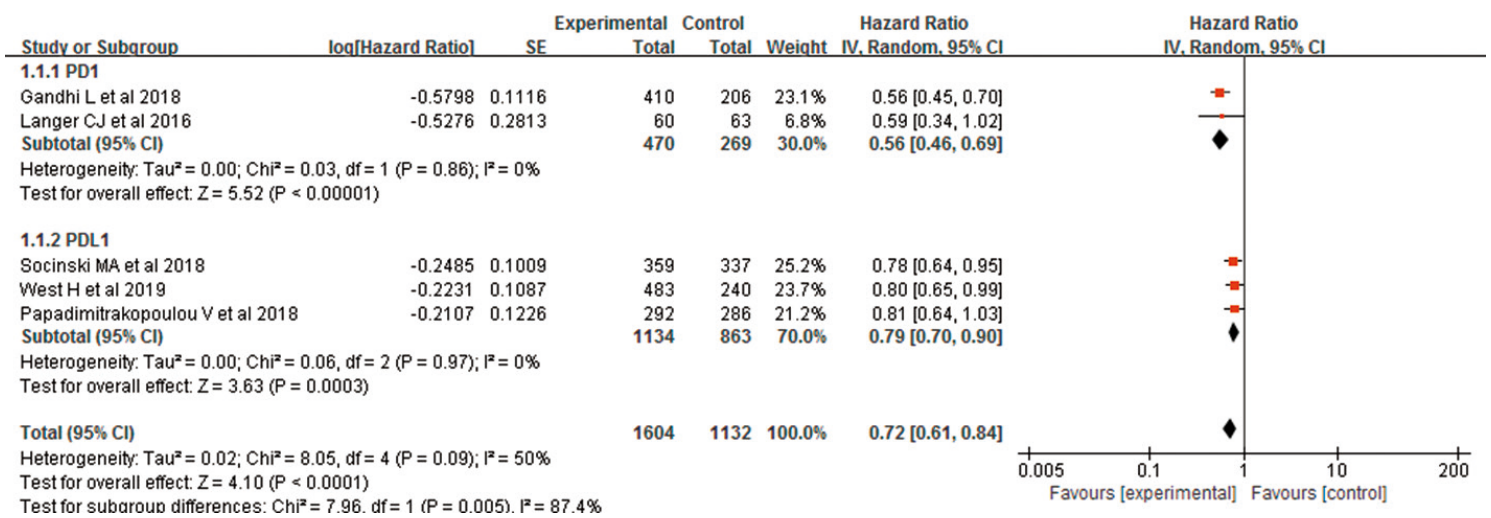

Figure 4 Forest plot of hazard ratios (HRs) for overall survival (OS).
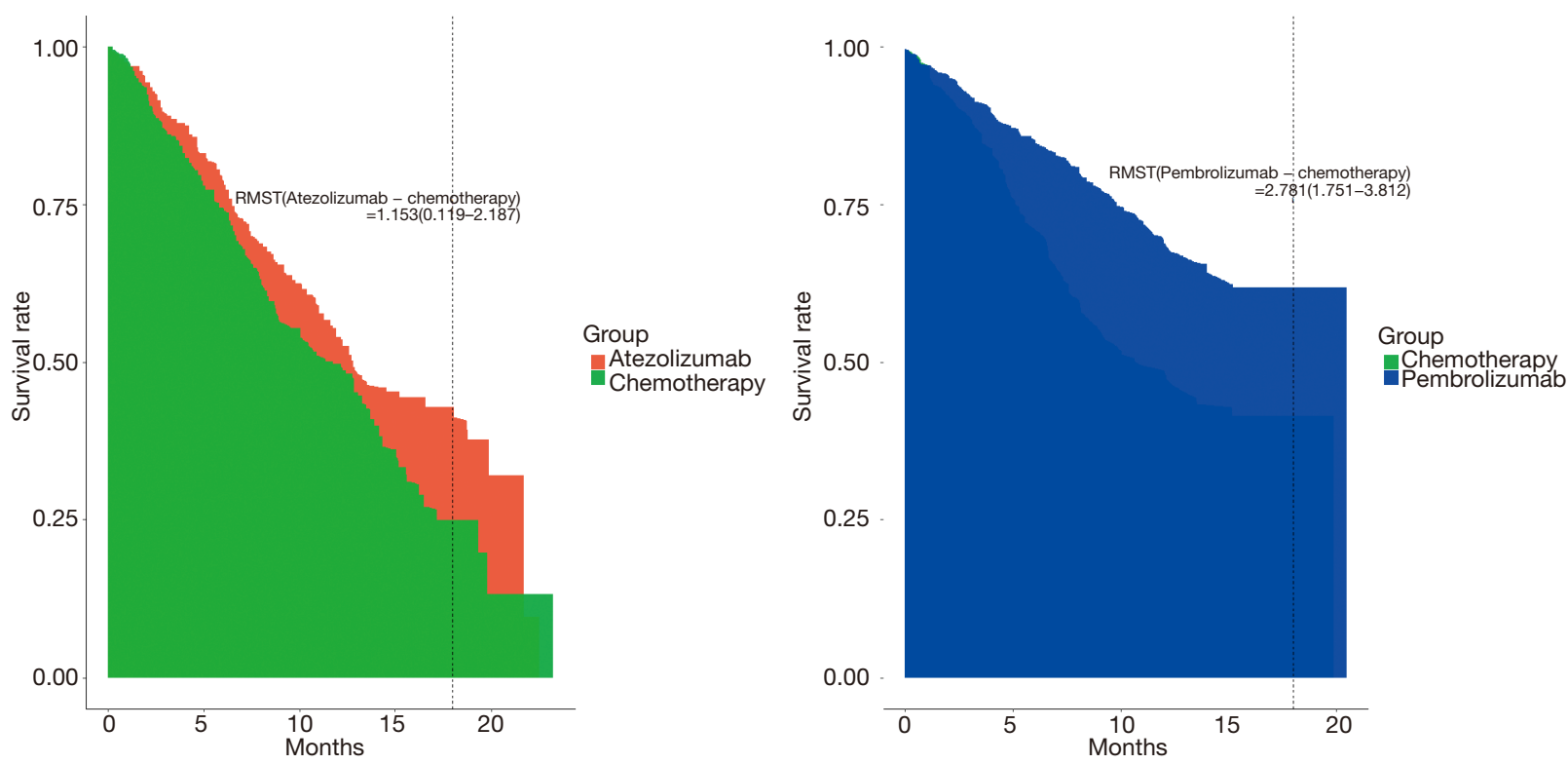

Figure 5 Differences in restricted mean survival time (RMST) at 18 months for the pembrolizumab + chemotherapy group and the atezolizumab + chemotherapy group compared with the chemotherapy group.

we focused on the combination of chemotherapy and ICIs in the treatment of non-squamous NSCLC. A significant lower risk of disease progression and death was found in both the PD-1 inhibitor + chemotherapy [PFS: 0.52 (0.44-0.62), $\mathrm{I}^{2}=0$; OS: $\left.0.63(0.49-0.81), \mathrm{I}^{2}=0\right]$ and PD-L1 inhibitor + chemotherapy groups [PFS: $0.61(0.56-0.68)$, $\mathrm{I}^{2}=0$; OS: $0.79(0.70-0.90), \mathrm{I}^{2}=0$ ] compared to conventional chemotherapy. Indirect comparison of the mature PFS data was conducted for pembrolizumab and atezolizumab based on 2 trials (KEYNOTE-189 and IMpower-132), and the results showed that the indirect HR was $0.87(0.67-1.13)$. Furthermore, an indirect comparison of RMST for OS was also conducted, which demonstrated a greater RMST in the pembrolizumab combination group than atezolizumab combination group [1.63 (0.17-3.09) months]. However, these results should be interpreted carefully, even though the assumption of similarity and consistency were taken into account, randomization did not hold across trials, and potential treatment-effect modifiers might bias results. Therefore, head-to-head clinical trials comparing pembrolizumab and atezolizumab are needed to determine optimal ICIs.

The high number of NSCLC cases have been attributed to tobacco smoking. This aetiological association and 
Table 2 Factors associated with PFS

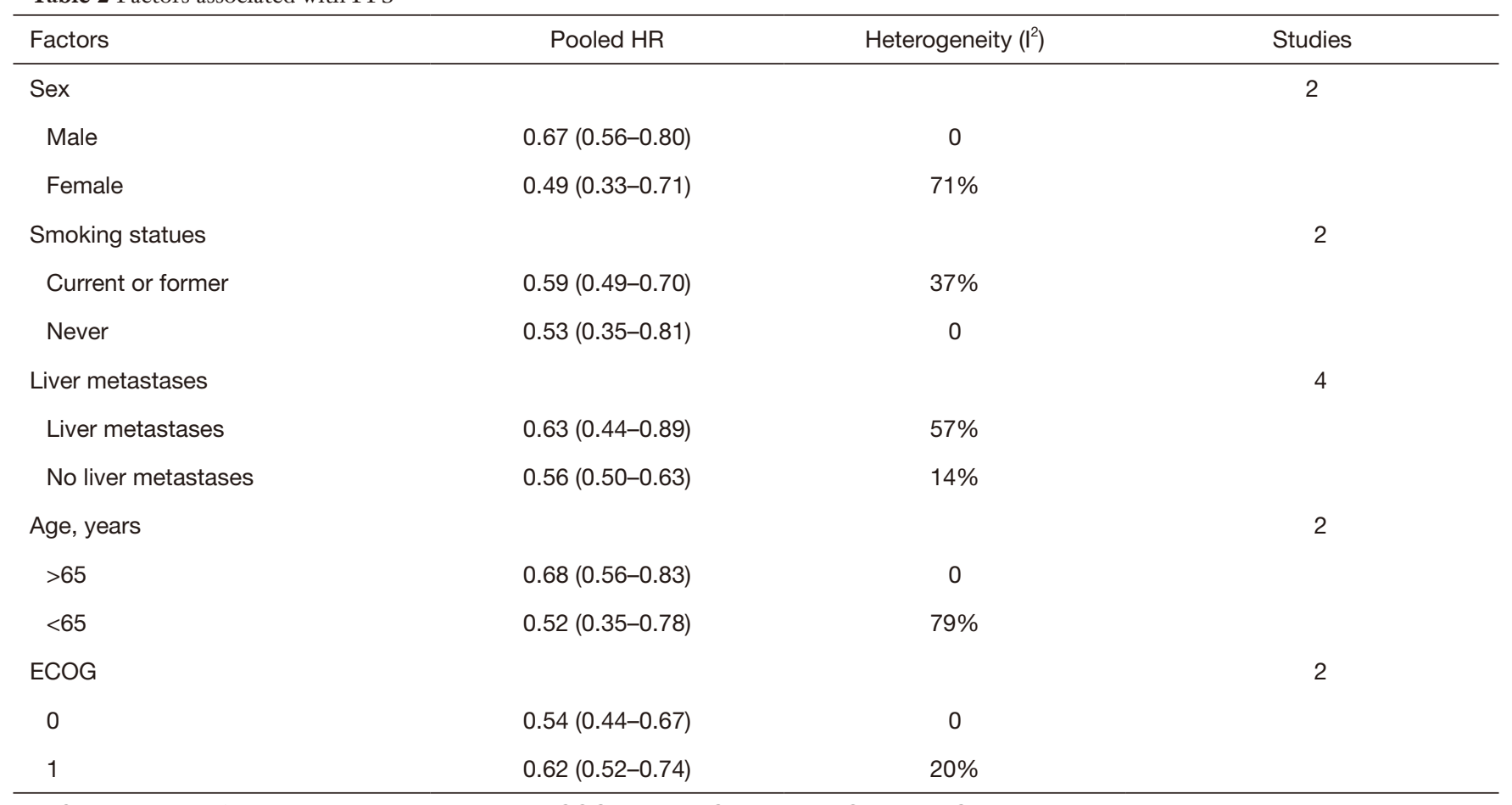

PFS, progression-free survival; HR, hazard ratio; ECOG, Eastern Cooperative Oncology Group.

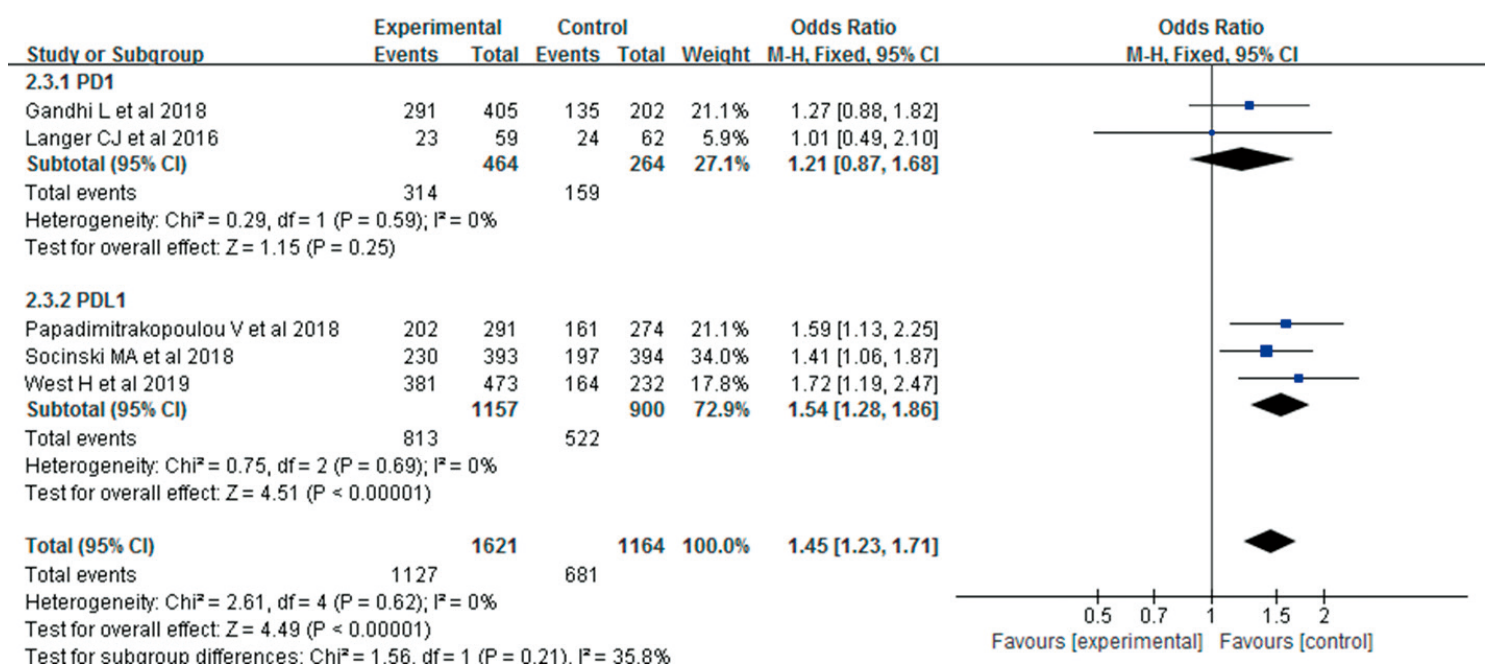

Figure 6 Forest plot of odd ratios (ORs) for any grade $\geq 3$ grade $\geq 3$ adverse events (AEs).

the somatic mutations generated by tobacco smoking are particularly relevant to immunotherapy for cancer (25). In correlation with immunogenicity, increased activity of ICIs has been observed in smoking-induced NSCLC (26). Due to the lower immunogenicity in NSCLC patients who have never smoked, the efficacy of ICIs is not better than current and former smokers with NSCLC (27). The present metaanalysis demonstrated that for non-squamous NSCLC patients that have never smoked, these patients could still benefit from the additional use of ICIs in combination with chemotherapy in terms of PFS $\left[0.53(0.35-0.81), \mathrm{I}^{2}=0\right]$, and that this might be attributed to the combination effects, as 
potential immunogenic effects were also discovered with conventional chemotherapy (28).

Previous reports have shown that the therapeutic benefit of ICIs in patients with liver metastases was restricted (29). In this study, we assumed that liver metastatic status might lead to a differential effect of ICIs combined with chemotherapy in the treatment of non-squamous NSCLC. As a result, significant PFS prolonging was seen in the subgroup of nonsquamous NSCLC patients with liver metastasis $[\mathrm{HR}=0.63(0.44-0.89)]$. However, heterogeneity between studies remains to be considered as $\mathrm{I}^{2}=57 \%$. Of the included 4 studies, 1 study explored the effect of pembrolizumab combined with chemotherapy [HR $=0.52(0.34-0.81)], 1$ study explored the effect of atezolizumab and bevacizumab combined with chemotherapy [HR $=0.42(0.26-0.67)]$, and the other 2 explored the effect of atezolizumab combined with chemotherapy [HR $=0.85(0.61-1.19)]$. Thus, the prolonging of PFS by atezolizumab for non-squamous NSCLC patients with liver metastasis was distinct. As the proportion of liver metastasis was small in the included trials, more large-scale real-world evidences is still needed to explored further and validate this phenomenon.

The potential of PD-L1 expression status as a bio-marker to guide molecular selection for non-squamous NSCLC was also explored in this study. The results showed that a benefit in PFS was found regardless of PD-L1 status. For patients with high PD-L1 expression $(>50 \%)$, the pooled HR for PFS was $0.41\left(0.33-0.52, I^{2}=0\right)$. For the intermediate PD-L1 expression (1-49\%) and negative PD-L1 expression $(<1 \%)$ subgroups, the pooled HR for PFS was 0.57 (0.47$\left.0.70, \mathrm{I}^{2}=0\right)$ and $0.75\left(0.64-0.86, \mathrm{I}^{2}=0\right)$. Importantly, the subgroup difference was significant $(\mathrm{P}<0.01)$, which may suggest that PD-L1 levels have predictive value for PFS benefit. However, the results should be interpreted carefully because of the inconsistent of evaluation methods used for the detection of PD-L1 protein levels in different studied (30), which might have introduced potential bias. Given this, standardized PD-L1 assay and evaluation methods and a uniform cut-off value, should be further discussed.

Nevertheless, our study still had some limitations. Firstly, this study was based on study-level evidence, the inclusion and exclusion criteria differences and the heterogeneity between studies might bias results. To minimize this limitation, we conducted subgroup analyses among treatment regimens. Additionally, the assessment of influencing factors and adverse events were mainly based on original studies without individual patient data, which might produce more reporting bias. Further large-scale real-world evidence is still needed to verify our results.

In conclusion, this meta-analysis confirmed the treatment effect of ICIs in combination with chemotherapy for nonsquamous NSCLC. The additional use of pembrolizumab demonstrated greater benefit for PFS and OS with no significant increase in AEs (> grade 3). Furthermore, a greater RMST was demonstrated in the pembrolizumab combination group compared with the atezolizumab combination group. For ICIs with atezolizumab, despite the benefit in PFS and OS, more attention should be paid to the significant increase in AEs (> grade 3). Overall, for non-squamous NSCLC, current data suggest a PFS advantage for non-squamous NSCLC using ICIs combined with chemotherapy, irrespective of PD-L1 expression level, smoking status, liver metastasis status, sex, age, and ECOG scores. As all trials were conducted in patients with nonsquamous NSCLC, these results' extrapolation is only valid in this population. To verify our results, further mature OS data is needed, and head-to-head clinical trials comparing pembrolizumab and atezolizumab are also needed to determine the treatment effects.

\section{Acknowledgments}

Funding: This work was supported by the CAMS Innovation Fund for Medical Sciences (CIFMS) (grants No. 2016-I2M1-001, 2017-I2M-1-005 and 2017-I2M-1-003).

\section{Footnote}

Reporting Checklist: The authors have completed the PRISMA reporting checklist. Available at http://dx.doi. org/10.21037/apm-20-1498

Conflicts of Interest: All authors have completed the ICMJE uniform disclosure form (available at http://dx.doi. org/10.21037/apm-20-1498). The authors report grants from CAMS Innovation Fund for Medical Sciences (grants No. 2016-I2M-1-001, 2017-I2M-1-005, and 2017-I2M-1003), during the conduct of the study.

Ethical Statement: The authors are accountable for all aspects of the work in ensuring that questions related to the accuracy or integrity of any part of the work are appropriately investigated and resolved.

Open Access Statement: This is an Open Access article 
distributed in accordance with the Creative Commons Attribution-NonCommercial-NoDerivs 4.0 International License (CC BY-NC-ND 4.0), which permits the noncommercial replication and distribution of the article with the strict proviso that no changes or edits are made and the original work is properly cited (including links to both the formal publication through the relevant DOI and the license). See: https://creativecommons.org/licenses/by-nc-nd/4.0

\section{References}

1. Bray F, Ferlay J, Soerjomataram I, et al. Global cancer statistics 2018: GLOBOCAN estimates of incidence and mortality worldwide for 36 cancers in 185 countries. CA Cancer J Clin 2018;68:394-424.

2. Travis WD. Pathology of Lung Cancer. Clin Chest Med 2011;32:669-92.

3. Planchard D, Popat S, Kerr K, et al. Metastatic non-small cell lung cancer: ESMO Clinical Practice Guidelines for diagnosis, treatment and follow-up. Ann Oncol 2018;29:iv192-237.

4. Pardoll DM. The blockade of immune checkpoints in cancer immunotherapy. Nat Rev Cancer 2012;12:252-64.

5. Garon EB, Rizvi NA, Hui R, et al. Pembrolizumab for the treatment of non-small-cell lung cancer. N Engl J Med 2015;372:2018-28.

6. Borghaei H, Paz-Ares L, Horn L, et al. Nivolumab versus Docetaxel in Advanced Nonsquamous Non-Small-Cell Lung Cancer. N Engl J Med 2015;373:1627-39.

7. Brahmer J, Reckamp KL, Baas P, et al. Nivolumab versus Docetaxel in Advanced Squamous-Cell Non-Small-Cell Lung Cancer. N Engl J Med 2015;373:123-35.

8. Fehrenbacher L, Spira A, Ballinger M, et al. Atezolizumab versus docetaxel for patients with previously treated non-small-cell lung cancer (POPLAR): a multicentre, open-label, phase 2 randomised controlled trial. Lancet 2016;387:1837-46.

9. Chen YM. Immune checkpoint inhibitors for nonsmall cell lung cancer treatment. J Chin Med Assoc 2017;80:7-14.

10. Galluzzi L, Buqué A, Kepp O, et al. Immunological Effects of Conventional Chemotherapy and Targeted Anticancer Agents. Cancer Cell 2015;28:690-714.

11. Apetoh L, Ladoire S, Coukos G, et al. Combining immunotherapy and anticancer agents: the right path to achieve cancer cure? Ann Oncol 2015;26:1813-23.

12. Peng J, Hamanishi J, Matsumura N, et al. Chemotherapy Induces Programmed Cell Death-Ligand 1 Overexpression via the Nuclear Factor- $\kappa \mathrm{B}$ to Foster an Immunosuppressive
Tumor Microenvironment in Ovarian Cancer. Cancer Res 2015;75:5034-45.

13. Langer CJ, Gadgeel SM, Borghaei H, et al. Carboplatin and pemetrexed with or without pembrolizumab for advanced, non-squamous non-small-cell lung cancer: a randomised, phase 2 cohort of the open-label KEYNOTE-021 study. Lancet Oncol 2016;17:1497-508.

14. Gandhi L, Rodríguez-Abreu D, Gadgeel S, et al. Pembrolizumab plus Chemotherapy in Metastatic NonSmall-Cell Lung Cancer. N Engl J Med 2018;378:2078-92.

15. Socinski MA, Jotte RM, Cappuzzo F, et al. Atezolizumab for First-Line Treatment of Metastatic Nonsquamous NSCLC. N Engl J Med 2018;378:2288-301.

16. Papadimitrakopoulou V, Cobo M, Bordoni R, et al. PFS and safety results with 1L atezolizumab + carboplatin/ cisplatin + pemetrexed in stage IV non-squamous NSCLC. J Thorac Oncol 2018;13:S332-3.

17. Cappuzzo F, Hussein M, et al. IMpower130: Progressionfree survival (PFS) and safety analysis from a randomised phase III study of carboplatin + nab-paclitaxel $(\mathrm{CnP})$ with or without atezolizumab (atezo) as first-line (1L) therapy in advanced non-squamous NSCLC. Ann Oncol 2018;29:viii742-3.

18. Huang B, Kuan PF. Comparison of the restricted mean survival time with the hazard ratio in superiority trials with a time-to-event end point. Pharm Stat 2018;17:202-13.

19. Uno H, Claggett B, Tian L, et al. Moving beyond the hazard ratio in quantifying the between-group difference in survival analysis. J Clin Oncol 2014;32:2380-5.

20. Rahmadian AP, Delos Santos S, Parshad S, et al. Quantifying the Survival Benefits of Oncology Drugs With a Focus on Immunotherapy Using Restricted Mean Survival Time. J Natl Compr Canc Netw 2020;18:278-85.

21. Moher D, Liberati A, Tetzlaff J, et al. Preferred reporting items for systematic reviews and meta-analyses: the PRISMA statement. PLoS Med 2009;6:e1000097.

22. Higgins JP, Altman DG, Gøtzsche PC, et al. The Cochrane Collaboration's tool for assessing risk of bias in randomised trials. BMJ 2011;343:d5928.

23. Zhang Y, Zhou H, Zhang L. Which is the optimal immunotherapy for advanced squamous non-small-cell lung cancer in combination with chemotherapy: anti-PD-1 or anti-PD-L1? J Immunother Cancer 2018;6:135.

24. Abdel-Rahman O, Elhalawani H. Risk of fatal pulmonary events in patients with advanced non-small-cell lung cancer treated with EGF receptor tyrosine kinase inhibitors: a comparative meta-analysis. Future Oncol 2015;11:1109-22.

25. Alexandrov LB, Nik-Zainal S, Wedge DC, et al. 
Signatures of mutational processes in human cancer. Nature 2013;500:415-21. Erratum in: Nature. 2013 Oct 10;502(7470):258. Imielinsk, Marcin [corrected to Imielinski, Marcin].

26. Chan TA, Yarchoan M, Jaffee E, et al. Development of tumor mutation burden as an immunotherapy biomarker: utility for the oncology clinic. Ann Oncol 2019;30:44-56.

27. Rizvi NA, Hellmann MD, Snyder A, et al. Cancer immunology. Mutational landscape determines sensitivity to PD-1 blockade in non-small cell lung cancer. Science

Cite this article as: Shi Y, Chen W, Li C, Zhang Y, Bo M, Qi S, Li G. Efficacy and safety of first-line treatments with immune checkpoint inhibitors plus chemotherapy for non-squamous non-small cell lung cancer: a meta-analysis and indirect comparison. Ann Palliat Med 2021;10(3):2766-2775. doi: 10.21037/ apm-20-1498
2015;348:124-8.

28. Chen DS, Mellman I. Oncology meets immunology: the cancer-immunity cycle. Immunity 2013;39:1-10.

29. Tumeh PC, Hellmann MD, Hamid O, et al. Liver Metastasis and Treatment Outcome with Anti-PD-1 Monoclonal Antibody in Patients with Melanoma and NSCLC. Cancer Immunol Res 2017;5:417-24.

30. Waqar SN, Morgensztern D. Immunotherapy for nonsmall cell lung cancer: are we on the cusp of a new era? Expert Rev Clin Immunol 2015;11:871-3. 